\title{
Does altitude moderate the impact of lithium on suicide? A spatial analysis of Austria
}

\author{
Marco Helbich $^{1 *}$, Victor Blüml2*, Michael Leitner ${ }^{3,4}$, Nestor D. Kapusta ${ }^{2}$ \\ ${ }^{1}$ Institute of Geography, University of Heidelberg, Heidelberg, Germany; ${ }^{2}$ Department of Psychoanalysis and \\ Psychotherapy, Medical University of Vienna, Vienna, Austria; ${ }^{3}$ Department of Geography and Anthropology, \\ Louisiana State University, Baton Rouge, USA; ${ }^{4}$ Department of Geoinformatics - Z_GIS, University of \\ Salzburg, Austria; "Both authors contributed equally to this research
}

\begin{abstract}
Suicide, the tenth leading cause of death worldwide, is a complex phenomenon. Models aiming to explain the interaction of ambient variables such as socioeconomic factors, lithium content of drinking water and altitude are poorly developed. While controlling for several risk factors, this research bridges two different, but complementary research lines by investigating statistically the relationship on suicide mortality between lithium levels in drinking water in response to altitude above sea level. Besides regression models with main effects, a multiplicative interaction model between lithium and altitude has been developed providing estimates at the district-level for Austria where spatial autocorrelation was accounted for through spatial filtering. The correlation results showed a negative association between lithium levels and altitude. The regression confirmed a negative association of lithium levels and suicide mortality. Altitude was found to be positively associated with suicide mortality. On the other hand, lithium effects on suicide mortality were found to be moderated by altitude. In lower altitude regions the effect turned out to be negatively related to suicide mortality, while lithium had a positive association in high-altitude regions. These results provide evidence for the fact that the relationship between lithium, altitude and suicide rates is more complex than hitherto assumed. Further research on the effects of ambient variables such as low levels of lithium on suicide is needed and particularly the lithium-altitude interaction is worth further investigation to understand possible underlying neurochemical processes.
\end{abstract}

Keywords: altitude, lithium in drinking water, regression, spatial filtering, suicide.

\section{Introduction}

Suicide is a major public health issue accounting for over one million deaths per year making it the tenth leading cause of death worldwide (Hawton and van Heeringen, 2009). It is a complex phenomenon with multiple risk factors including age, sex, ethnic origin, marital status, psychiatric disorders, availability of psychosocial services and other sociodemographic characteristics such as religious denomination and unemployment (Mann et al., 2005; Hawton and van Heeringen, 2009). Suicide rates vary greatly between countries and also between different geographical regions within countries (e.g. Kapusta et al., 2011; Helbich et al., 2012) but the underlying reasons are not well understood (Mann et al., 2005; Hawton and van Heeringen, 2009).

Recent research has found evidence that lithium levels in drinking water supply may have an impact on

Corresponding author:

Marco Helbich

Institute of Geography, University of Heidelberg

Berliner Strasse 48, D-69120 Heidelberg, Germany

Tel. +496221545 572; Fax +496221544529

E-mail: helbich@uni-heidelberg.de the regional distribution of suicide mortality rates. Higher levels of lithium have been associated with lower suicide rates (Schrauzer and Shrestha, 1990; Ohgami et al., 2009; Kapusta et al., 2011; Helbich et al., 2012; Blüml et al., 2013). While suicide protective properties of lithium in therapeutic dosages are well established (Baldessarini et al., 2006; Guzzetta et al., 2007), the exact mode of action of the low levels of lithium found in drinking water is still not known. Indeed, contradictory results have been reported (Kabacs et al., 2011) and the few conducted studies have been challenged due to limitations such as too simplistic statistical models, disregard of the possible influence of space as well as potential interaction effects (Huthwaite and Stanley, 2010; Yang, 2011). In this context, spatial analysis is beneficial (Richardson et al., 2013). Of particular relevance is the consideration of spatial effects in statistical regression models (Helbich et al., 2012).

Altitude has been reported to influence suicide rates (Brenner et al., 2011; Kim et al., 2011). In the United States of America (USA), it has been found to be an independent risk factor for suicide even when controlling for age, sex, race, median household income, gun ownership, and population density (Brenner et al., 
2011; Kim et al., 2011). It was speculated that in higher altitude regions, metabolic stress with mild hypoxia might lead to a worsening of mood disorder symptoms and thus to higher suicide rates (Brenner et al., 2011; Kim et al., 2011). These findings have been criticised for not controlling for possible confounding factors such as sociodemographic variables and the availability of mental health care, alleged to be a more plausible explanation for higher suicide rates in high-altitude regions (Betz et al., 2011, 2012). Nevertheless, altitude is also known to have an effect on the pharmacokinetics and has been shown to significantly influence lithium pharmacokinetics in healthy humans (Arancibia et al., 2003). These changes were considered clinically relevant and there is indeed a report of potential altitude-induced hypomania in a bipolar adolescent person secondary to altitude-related changes in lithium pharmacokinetics (Brahm and Puls, 2011). Lithium, a soluble alkali metal, is dissolved by rain water from rock and soil and is therefore, depending on the prevailing type of rock, found in water in certain areas (Schrauzer, 2002). Moreover, the lithium concentrations in ground water have been reported to be dependent on altitude (Shvartsev, 2008).

Based on the brief literature review, it can be concluded that (i) both lithium and altitude have an impact on suicide mortality and (ii) that a combined lithium-altitude interaction seems rational, although empirical evidence is lacking thus far. It was hypothesised that the recently found influence of altitude on suicide mortality rates might be due to differences in lithium concentrations, both in ground and drinking water at different altitudes. Furthermore, it was also hypothesised that altitude might have a moderating effect on the influence of lithium in drinking water on suicide rates, which has not been accounted for in previous research. Therefore, the present research was initiated with the aim to examine the association between lithium, altitude and suicide mortality in more detail by using advanced statistical methodology to account for possible spatial autocorrelation by means of spatial filtering.

\section{Materials and methods}

\section{Study site and data}

This study was conducted in Austria with the district level serving as the basic unit of analysis. The full set of all districts were considered $(\mathrm{N}=99)$. The initial dataset comes from Kapusta et al. (2011), with several variables being added for this present research.
Suicide data for the period between 2005 and 2009 were collected from the official Austrian mortality database of Statistics Austria. To account for different age distributions at the district level, the originally collected suicide data were transformed and expressed as standardised mortality ratios (SMRs) for suicide by taking the age composition of the general Austrian population as standard. Subsequently, the SMRs represent the response variable and were mapped in the top panel of Fig. 1. Above average SMRs (mean 0.802; standard deviation (SD) 0.198) can be observed in the alpine areas $(>0.985)$, while the eastern parts of Austria outside the alpine areas show relatively low SMRs (<0.719) (see Fig. 1).

The present research focused on lithium content in drinking water and altitude as the two covariates of main interest. Information about the former, obtained from AQA GmbH, covers the period from 2005 until the fall 2010. On the basis of in-situ measurements, lithium content values were taken from a total of 6,460 water samples from various places in the districts. The lowest measurable threshold lithium level, determined by inductively coupled plasma optical emission spectrometry, was $0.0033 \mathrm{mg} / \mathrm{l}$. The individual lithium measurements were aggregated at the district level (mean $0.010 \mathrm{mg} / \mathrm{l}$; SD $0.011 \mathrm{mg} / \mathrm{l}$ ) for statistical analysis. On average, 65.3 samples were collected from each district with a range from 1 to 312. In terms of lithium levels (Fig. 1, middle panel) an eastwest trend is apparent, with the eastern areas showing considerably higher lithium levels (above $0.016 \mathrm{mg} / \mathrm{l}$ ) compared to western Austria with $0.004 \mathrm{mg} / \mathrm{l}$.

A digital elevation model (DEM) was processed to derive the average altitude for each Austrian district. The DEM was provided by the Department of Geography and Regional Research at the University of Vienna (Federal Office of Metrology and Surveying, 2007). It represents a nationwide dataset with a spatial resolution of $10 \mathrm{~m}$. The average altitude information for each district was calculated with basic geographical information system (GIS) procedures, applying zonal statistics. Fig. 1 (lower panel) depicts the spatial distribution of the districts' altitude. The average altitude is approximately 726 $\mathrm{m}$ above the mean sea level (SD $485 \mathrm{~m}$ ) with the highest values being recorded in the southern and western districts. A visual comparison between the lithium content (middle panel) and the altitude map (lower panel), points to a negative correlation between the two variables, i.e. districts at higher altitudes tend to be associated with lower lithium levels and vice versa. 


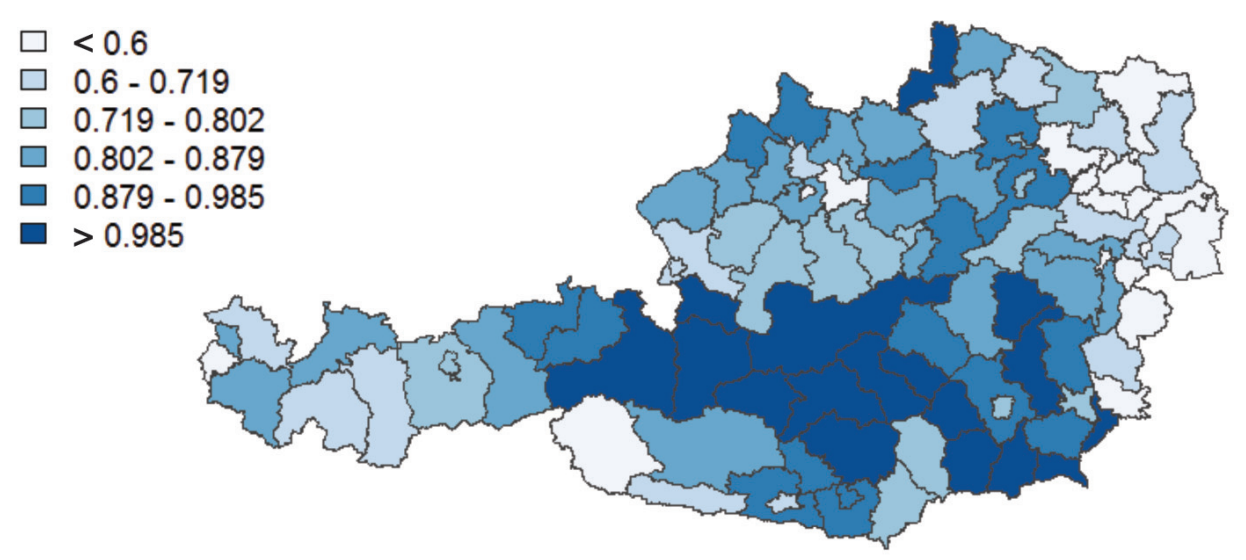

a
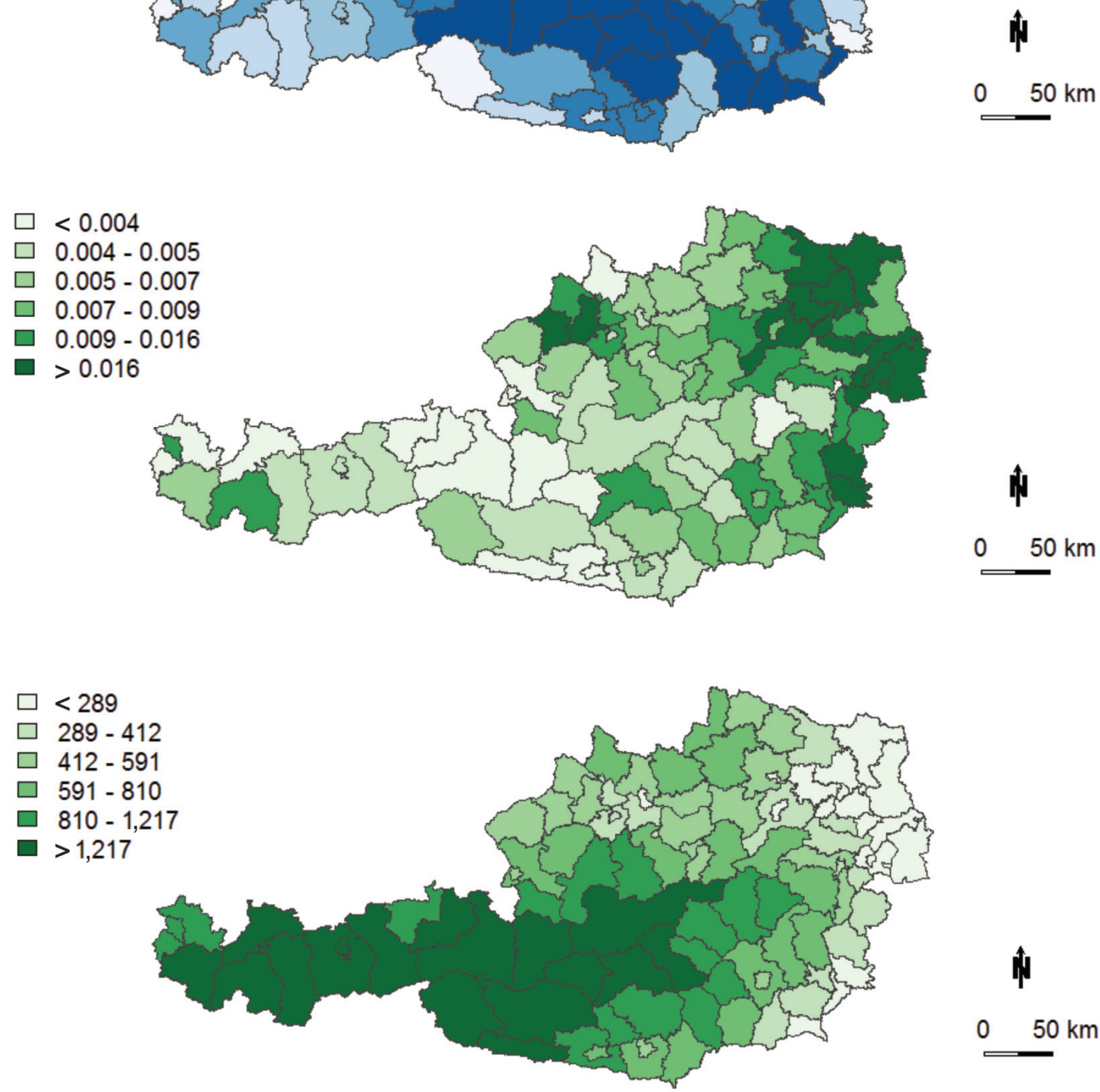

Fig. 1. Spatial distribution of: (a) SMRs (top panel); (b) lithium levels (mg/l); and (c) altitude (meters).

In addition to investigating the possible effects on SMR from lithium content in drinking water and altitude, seven control variables were collected (source within parantheses). These were (i) the population density for the year 2001 (Statistics Austria); (ii) the average income per capita for the year 2001 (Statistics Austria); (iii) the proportion of Roman Catholics for the year 2001 (Statistics Austria); (iv) the number of psychiatrists per 10,000 population for the year 2007
(Austrian Medical Chamber); (v) the density of general practitioners for each district for the year 2007 (Austrian Medical Chamber); (vi) the density of psychotherapists per 10,000 for the year 2005 (Austrian Institute of Health); and (vii) the average unemployment rates for 2005-2008 (Austrian Public Employment Service). Table 1 reports detailed descriptive statistics for SMR and for each of the nine covariates used in this research. 
Table 1. Descriptive statistics of variables included in this study.

\begin{tabular}{llrrrrr}
\hline Variable & Acronym & $1^{\text {st }}$ quartile & Median & Mean & $3^{\text {st }}$ quartile & SD \\
\hline Standardized mortality ratio & SMR & 0.671 & 0.802 & 0.790 & 0.901 & 0.198 \\
Lithium level, mean (mg/l) & LITH & 0.005 & 0.007 & 0.010 & 0.012 & 0.011 \\
Mean district altitude (m above the see level) & ALTD & 335 & 591 & 726 & 1,027 & 485 \\
Population density in 2001 (per km²) & POPD & 50.2 & 74.3 & 239.4 & 118.7 & 528.3 \\
Per capita income in 2001 (1,000 Euro) & INCO & 13.230 & 14.100 & 14.620 & 15.700 & 1.968 \\
Proportion of Roman Catholics in 2001 (\%) & ROMC & 75.4 & 82.6 & 80.9 & 90.3 & 10.7 \\
Psychiatrist density in 2005 (per 10,000) & PSYC & 0.350 & 0.520 & 0.798 & 0.975 & 0.720 \\
Psychotherapist density in 2007 (per 10,000) & PSTH & 1.615 & 2.360 & 4.088 & 3.915 & 5.260 \\
General practitioner density in 2007 (per 10,000) & GPRD & 6.405 & 7.410 & 7.581 & 8.235 & 1.658 \\
Unemployment rate in 2005-2008 (\%) & UNEMP & 1.838 & 2.325 & 2.349 & 2.688 & 0.731 \\
\hline
\end{tabular}

\section{Modelling}

The modelling part in this paper comprises of two main components. At first, a linear regression model with interaction effects was formulated. Due to the bias of spatial effects observed in the original regression model, it was extended by eigenvector filtering.

Regression model. Previous studies testing the association between SMR and lithium, possibly moderated by altitude, utilised multiple ordinary least square (OLS) regression analysis (Kim et al., 2011). In contrast to, for example, Kapusta et al. (2011), our study does not only focus on additive effects, but also includes a conditional (moderation) effect of the impact of lithium on SMR through the "altitude" covariate. Within the regression framework, conditional effects were modelled by means of interaction terms, represented as the product of two covariates (Jaccard et al., 1990, Brambor et al., 2006, Berry et al., 2012). In the course of modelling interaction effects, Brambor et al. (2006) state that a lack of multicollinearity among the variables is critical and that there is a need to pay special attention to model validation, e.g. by variance inflation factors (VIFs) as done by Fox (2002). A violation of this assumption increases standard errors. It should also be noted that the interpretation of the coefficient differs in comparison to a model with additive terms, when interaction effects $(\mathrm{X} \times \mathrm{Z})$ are included. Considering a conditional effect, the marginal effect of the variable $X$ (given by $\beta_{x}+\beta_{x z} Z$ ) is conditional on $Z$. If $Z=0$ then the marginal effect of $X$ on $Y$ reduces to $\beta_{\mathrm{x}}$. Due to the constant slope $\beta_{\mathrm{xz}}$ the marginal effect of $X$ changes by $\beta_{x z}$ for every unit increase in $Z$ (Berry et al., 2012).
Spatial filtering. As noted by Kim et al. (2011) and shown by Helbich et al. (2012), suicide rates are subject to regional variations, which violate OLS model assumptions. For example, such regional variations imply that a district has a similar SMR as an adjacent district, but a rather different SMR than district located further away. The spatial phenomenon of similar values being located nearby or clustered in space is termed (positive) spatial autocorrelation (SAC). Ignoring such a spatial process, as observed by Kim et al. (2011) and Kapusta et al. (2011), has consequences for classical statistical inference, including biased and inconsistent parameter estimates and/or standard errors, which may cause invalid conclusions as discussed by Anselin and Bera (1998). It is thus of paramount importance to model SAC explicitly.

As has been proven by Thayn and Simanis (2013), spatial eigenvector filtering (SEF) (Griffith, 2000; Tiefelsdorf and Griffith, 2007) is such a powerful and flexible approach to explicitly model SAC in regressions. SEF is based on the decomposition of the Moran's coefficient (MC) for a given spatial weight matrix, describing the spatial configuration of districts. The MC is a spatial statistic to test whether a spatial pattern includes SAC. The range of the MC is between -1 and +1 . Negative values indicate that neighbours are dissimilar to each other, while positive values indicate the spatial clustering of high, medium or low values, and a value around 0 represents spatial randomness. Henceforth, following Patuelli et al. (2011), the queen neighbourhood schema coupled with a coding, which refers to a global standardization of the weight matrix, was employed. Eigenvector decomposition was utilised to extract a set of orthogonal and independent eigenvectors (EVs) directly from 
this matrix. Tiefelsdorf and Boots (1995) have shown that each EV portrays a certain degree of latent SAC. For example, the first EV reflects the largest possible $\mathrm{MC}$ value, while the second EV expresses the largest obtainable MC by any possible set of EVs that is not correlated with the first one. Typically, the first few EVs mimic more global trends, while higher order EVs imitate regional to local patterns (Griffith, 2000). To initially reduce the EVs to a manageable set of candidate EVs, Tiefelsdorf and Griffith (2007) propose to focus on EVs above the threshold value of $\mathrm{MC} / \mathrm{MC}_{\max }$ $>0.25$. Through a stepwise variable selection algorithm by minimizing the Akaike information criterion (AIC) score, the response variable SMR is regressed onto these candidate EVs which results in the final set of significant EVs. Lastly, to achieve a more parsimonious spatial filter (SF), a linear combination was computed (Grimpe and Patuelli, 2011). This SF is included as a supplementary explanatory variable in the linear model and serves as surrogate for possible missing predictors, simultaneously absorbing SAC effects.

After sequential model testing, this research estimates the following moderated spatially filtered OLS model:

$$
\begin{gathered}
\mathrm{SMR}_{i}=\beta_{0}-\beta_{1} \mathrm{LITH}_{i}+\beta_{2} A L T_{i}+ \\
\beta_{3}\left(L I T H_{i} \times A L T_{i}\right)+\sum_{k=1}^{7} \beta_{k} C V_{k, i}+\sum_{l=1}^{l} \beta_{l} E V_{l, i}+\varepsilon_{i}
\end{gathered}
$$

where $S M R_{i}$ is the standardised mortality ratio in district $i$, the $\beta$ s are the coefficients to be estimated, the multiplicative term $L I T H_{i} \times A L T_{i}$ is the interaction effect between lithium and altitude, $C V_{k}$ is the $k$ th control variables, the $l$ th $E V_{l}$ are the selected spatial filters, and $\varepsilon$ is the error term. The inclusion of spatial filtering turns the basic, non-spatial regression model into a spatially explicit model, which is of crucial importance when analysing spatial data.

\section{Results}

Initially, the relationship between the SMR and both covariates lithium content in drinking water and altitude was analysed by non-parametric Spearman's rank correlation coefficients. As expected, both covariates showed a significant correlation with SMR. While lithium is negatively related to $\operatorname{SMR}(\rho=-0.26$; $\mathrm{P}<0.01$ ), altitude showed a positive association $(\rho=0.45 ; P<0.001)$. Moreover, the hypothesised relation between lithium and altitude was confirmed by a significant and negative correlation of $\rho=-0.64$ $(\mathrm{P}<0.001)$.
As SAC effects are critical for the subsequent regression analysis, all variables were tested by means of the MC. Using the queen contiguity condition coupled with the globally standardised weight matrix coding, the MC turned out to be highly significant $(\mathrm{P}<0.001)$ for all variables, with the exception of population density, psychiatrist density, general practitioner density and unemployment rate, which were found to be randomly distributed across space $(\mathrm{P}>0.05)$. These results support the notion that space might have a substantial impact on regression analysis, which possibly demands spatial filtering.

In the next step, the individual, additive effects of lithium level and altitude were tested in a non-spatial multiple regression model (model 1), where SMR was regressed on the set of all covariates included in Table 1. As indicated by the exploratory analysis through MCs, validation of the model assumptions is in this case of utmost importance. The results show that this model is severely affected by spatially non-independent residuals $(\mathrm{MC}=0.194 ; \mathrm{P}<0.001)$, biasing the estimations of the model. Additionally, heteroskedasticity turned out to be a problem $(\mathrm{BP}=20.09, \mathrm{P}<0.05)$. Finally, an adjusted $R^{2}$ of 0.323 points to a low model performance. Due to its misspecification, this initial model will not be further discussed here.

One solution to gain reliable estimates is to absorb the present SAC effects by incorporating a SF into the initial model. Based on the selected neighbourhood specification (queen contiguity and C-coding; see Patuelli et al., 2011), 22 candidate EVs had a MC larger than the threshold of 0.25 . To further reduce the set of EVs, SMR was regressed onto these $22 \mathrm{EV}$ s to select the significantly associated final EVs. By computing the linear combination of those seven resulting EVs established the final SF, which turned out to explain approximately $32 \%$ of the SMR variance. Clearly, this emphasises the importance of space in statistical analysis and supports the conclusions in Helbich et al. (2012). Therefore, the aspatial model 1 was extended by this SF and re-estimated (model 2 in Table 2). With an adjusted $R^{2}$ increase and AIC decrease, the fit of model 2 was significantly improved. To eliminate nonsignificant covariates, a stepwise covariate selection by means of AIC reduction was applied resulting in model 3. The AIC was further reduced to -108 and $53 \%$ of the variance could now be explained. Additionally, the SF was significant and effectively absorbed the SAC, which was confirmed by a none significant MC $(\mathrm{P}=0.933)$. Model 3 was also wellbehaved, because heteroskedasticity was rejected $(\mathrm{BP}=$ 8.04; $\mathrm{P}=0.154)$, residuals were normally distributed 


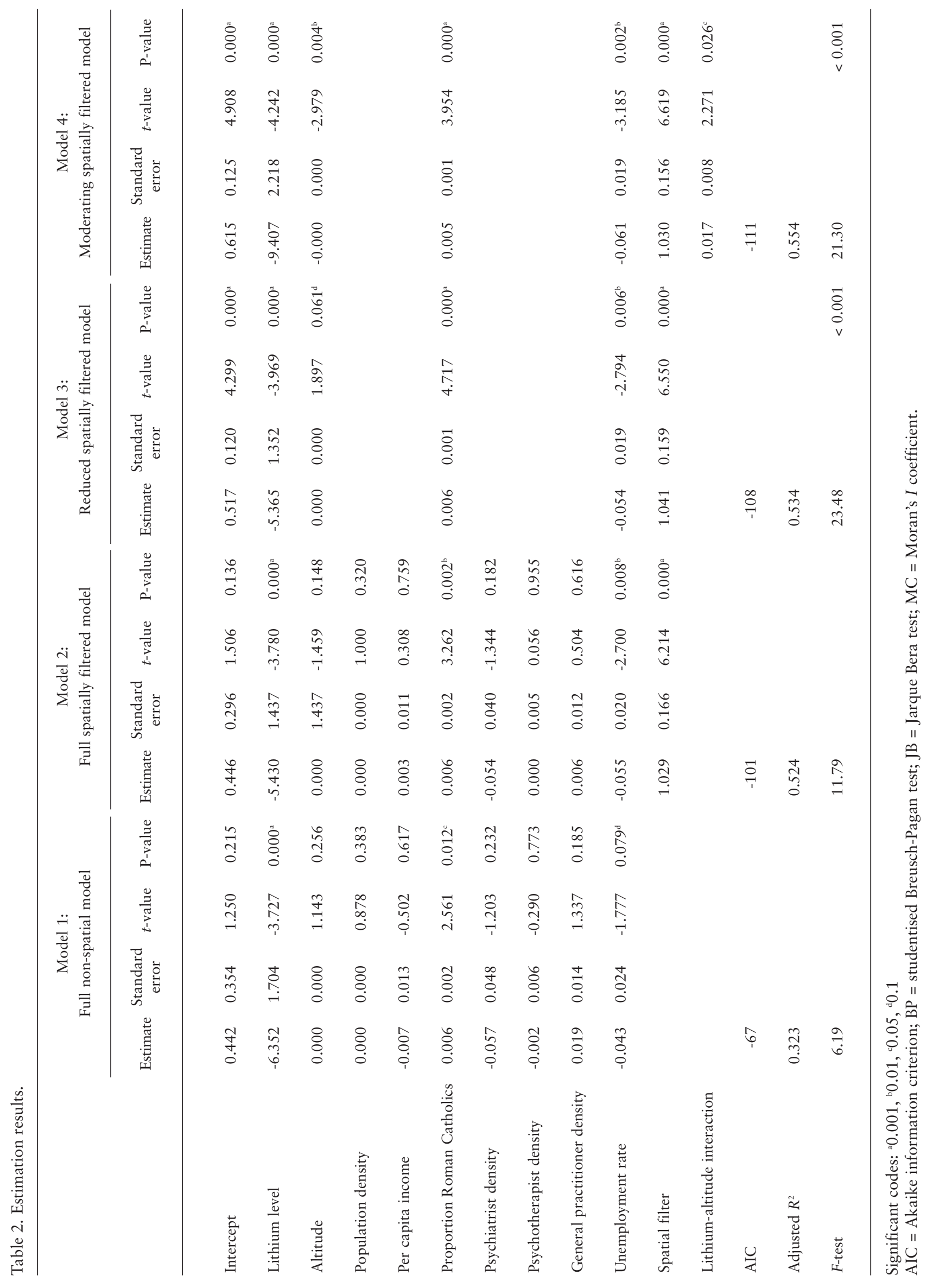


$(\mathrm{JB}=1.541 ; \mathrm{P}=0.463)$ and the RESET test detected no misspecification of the functional form $(\mathrm{P}=0.281)$. The results indicate that lithium level, proportion of Roman Catholics and unemployment rate are significant predictors of SMR at the $95 \%$ significance level and altitude at the $90 \%$ significance level. More specifically, a statistically significant negative relationship between lithium content in drinking water and SMR is found. One unit increase in lithium yields approximately a fivefold decrease in the suicide SMR. In contrast, altitude is positively associated with suicide SMR. The other significant control variables showed expected signs.

Above and previously published models are limited by considering lithium level and altitude as additive effects. Therefore, the final model 4 extends model 3 by an interaction effect between these two covariates (Table 2). The results show that model 4 performs better than model 3 due to an AIC score of -111 and an adjusted $R^{2}$ value of 0.554 . Also an ANOVA test clearly favours model 4 by rejecting the hypothesis that the interaction effect does not add significant explanatory power $(F$-test $=5.16 ; \mathrm{P}=0.025)$. As discussed above, multicollinearity may be problematic due to the inclusion of the interaction parameter into model 4 . However, this can be rejected, since for all variables the VIF scores were below 3.8, i.e. clearly below the critical value of 10 recommended by Fox (2002). None of the statistics employed to verify model assumptions depicted any anomaly.

The coefficient for lithium was found to be negative and significant. This indicates that higher lithium levels in the drinking water would decrease the SMR. At sea-level (the altitude equals zero), a one unit increase in lithium would reduce the SMR about ninefold. Similarly, if lithium equals 0 the weaker main effect of altitude on the SMR is statistically significant and negative. Considering the interaction effect, the magnitude of the estimated coefficient for lithium was less pronounced. It must be noted that the models' coefficients in model 4 cannot be directly compared to the previous models 1 to 3 . The existence of these twoway interaction effects was verified through the significance of a product term at the 95\% level and showing a positive sign. There is thus significant evidence that the impact of lithium on the SMR varies with altitude. When compared to the results in Helbich et al. (2012), the lithium effect was less pronounced at the mean altitude when an additional interaction effect was included. Because such higher-order model terms are difficult to interpret, the so-called effect displays introduced by Fox (2003) were applied to increase the quality of the interpretation. Effect displays visualise statistical terms in regression models, where a covari-

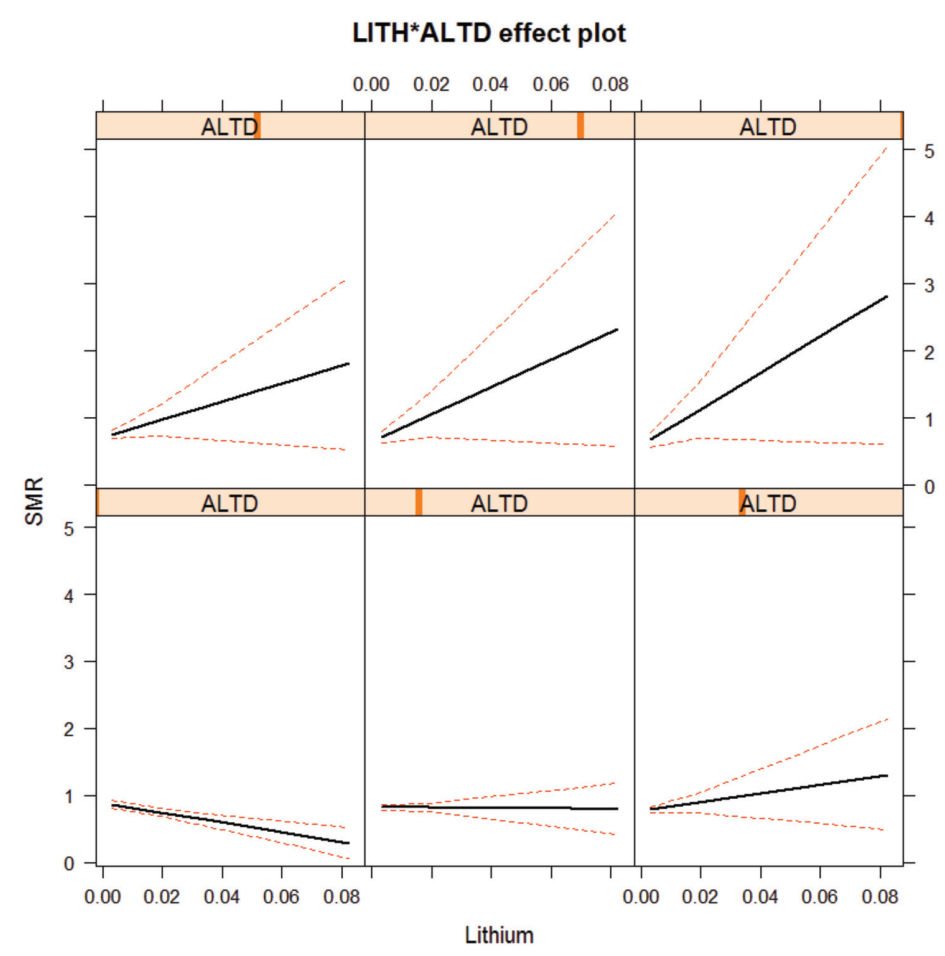

Fig. 2. Effect display for the lithium-altitude interaction. Solid lines show the marginal effect, while the dashed lines represent the $95 \%$ confidence envelopes around the fitted values. 
ate is allowed to vary across a set of values of a moderating variable, while other covariates are being held constant at a typical value such as the mean. Fig. 2 shows the marginal effect and the corresponding 95\% confidence envelopes. To illustrate how the marginal effect of lithium changes, altitude was varied between the $10^{\text {th }}$ and the $90^{\text {th }}$ percentile, which clearly reveals that the impact of lithium depends on the altitude level. While the effect of lithium on SMR was slightly negative in lower altitudes (lower left panel), the sign changed to positive for higher altitudes and became more pronounced, while the remaining covariates were held constant at their sample means. There seemed to be no effect for medium altitudes. Simultaneously, due to fewer sample data for higher altitude, the $95 \%$ confidence intervals (CIs) increased. This indicates that the effects are less precisely estimated for higher altitudes. Moreover, and in contrast to Helbich et al. (2012), no statistical support was found that psychiatrist density had a significant effect on suicide SMR. Similarly, the effect that the proportion of Roman Catholics had on the suicide SMR again matched the results from Helbich et al. (2012). Finally, unemployment rate had a significant negative association with suicide SMR.

The final step in the analysis of model 4 decomposes the $R^{2}$ to determine the relative importance of each covariate using the sequential sums of squares method (LGM) (Grömping, 2009). To receive the 95\% CI level, 1,000 bootstrap runs were employed. The contribution of each covariate is depicted in Fig. 3. Not surprisingly, with a relative importance of approximately $40 \%$, the SF was found to be the most important covariate, while lithium and the proportion of Roman

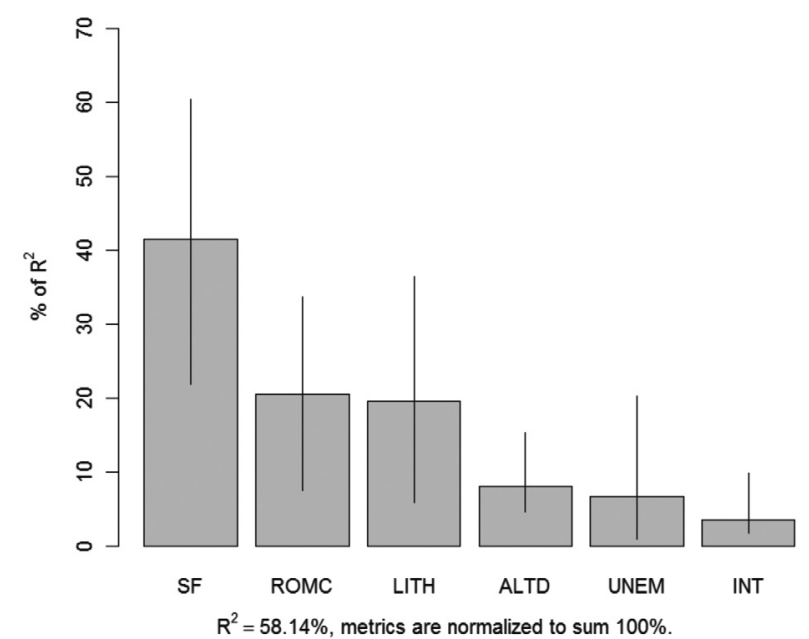

Fig. 3. Relative covariate importance for SMR with $95 \%$ bootstrap confidence intervals using the LMG method.
Catholics turned out to be the second most important covariates, each contributing approximately $20 \%$ to the overall $R^{2}$. The two covariates, altitude and unemployment rate, were found to be less important as they provide only $8 \%$ and $7 \%$, respectively to the overall $R^{2}$. With about $4 \%$ contribution of the interaction effect (INT), its significance was rather small.

\section{Discussion and conclusions}

The present research investigated statistically the impact of lithium in drinking water on suicide in response to altitude from 99 districts located in Austria from a spatial perspective. Simple correlation analysis showed a distinct positive association between altitude and SMR thus corroborating previous studies (Brenner et al., 2011; Kim et al., 2011) speculating that the influence of altitude on brain oxygen supply might explain higher suicide rates in high altitude areas (Brenner et al., 2011; Kim et al., 2011). Supporting Shvartsev's findings (2008), which demonstrated that the general salinity of ground waters increases with decreasing altitude, an association which holds true for lithium, our results also showed a negative association between lithium concentrations and altitude. Considering the previously reported negative association between lithium levels in the drinking water and SMR by Kapusta et al. (2011) and Helbich et al. (2012), these findings offer a novel, possible explanation for the reported association between altitude and SMR insofar as lower lithium concentrations in the ground and the drinking water might be responsible for higher suicide rates in high-altitude areas. Of course, this hypothesis needs further scrupulous examination.

Subsequently, our study expands previous simple main effects models, testing only the effect of lithium on suicide in addition to other control variables, by including the predictor altitude as well as its interaction effect with lithium. The significant negative association of lithium and suicide rates in Austria still held true. More importantly, this study is able to demonstrate that this effect is moderated through altitude. While lithium is negatively associated with suicide rates in lower altitude regions, this association changes to a positive correlation in high-altitude regions. The modelling results show that simply assuming additivity in the parameters, as in Helbich et al. (2012) and others, hides essential relationships, and that more complex associations can be expected. The possible mechanism of action of this moderating effect of altitude on lithium activity is uncertain. It has been reported that altitude influences the pharmacokinetics of lithium and 
that these changes might be clinically significant (Arancibia et al., 2003; Brahm and Puls, 2011). Yet, more research using experimental designs is needed to investigate the interaction between lithium and altitude in more detail. Finally, the results highlight the requirement for an explicit consideration of space in this type of medical research. To our knowledge, this paper successfully uses SEF for the first time in medicine to correct for spatial effects in linear models.

Although the present research yielded several theoretically and methodologically important results, the proposed model disregards possible non-linear relationships. Future work will thus have to explore nonparametric or semi-parametric non-linear models. Furthermore, future models must take into account the hierarchical nature of lithium data, which are sampled at certain geospatial locations. In accordance to the modifiable areal unit problem, this aggregation process at the district level may induce some artificial influence on the estimated parameters. Although our well behaved model refutes this argumentation. Moreover, one limitation of the dataset is the relatively few collected data points for higher altitudes. Therefore, the results presented in this research should be taken with some caution and require replications in other regions, ideally applying a larger and more homogenous sample than compared to the data set used in this study, before plausible and holistic conclusions for medical research can be drawn. Despite of these future improvements, this study sheds new light on the relationship that lithium has with SMR. The results provide clear evidence that this impact is noticeably more complex than hitherto assumed insofar as the effect of lithium is additionally moderated by altitude. We finally argue that altitude effects on suicide mortality seem to be a function of lithium contents of drinking water.

\section{Acknowledgements}

We gratefully acknowledge Doris and Andreas Riedl at the Department of Geography and Regional Research at the University of Vienna for providing us with the digital elevation model used in this research. The research was funded by the Austrian Science Fund (FWF) through the Doctoral College GIScience (DK W 1237-N23). The authors have no competing financial interests.

\section{References}

Anselin L, Bera A, 1998. Spatial dependence in linear regression models with an introduction to spatial econometrics. In: handbook of applied economic statistics. Ullah A, Giles D, (eds).
Marcel Dekker, New York, 237-289 pp.

Arancibia A, Paulos C, Chavez J, Ritschel WA, 2003. Pharmacokinetics of lithium in healthy volunteers after exposure to high altitude. Int J Clin Pharm Th 41, 200-206.

Baldessarini RJ, Tondo L, Davis P, Pompili M, Goodwin FK, Hennen J, 2006. Decreased risk of suicides and attempts during long-term lithium treatment: a meta-analytic review. Bipolar Disord 8, 625-639.

Berry WD, Golder M, Milton D, 2012. Improving tests of theories positing interaction. J Polit 74, 653-671.

Betz M, Valley MA, Lowenstein SR, Hedegaard H, Thomas D, Stallones L, Honigman B, 2011. Elevated suicide rates at high altitude: sociodemographic and health issues may be to blame. Suicide Life Threat Behav 41, 562-573.

Betz M, Valley MA, Lowenstein SR, Honigman B, 2012. Suicide at high altitude: the importance of sociodemographic and mental health care factors. High Alt Med Bio 13, 138.

Blüml V, Regier MD, Hlavin G, Rockett IRH, König F, Vyssoki B, Bschor T, Kapusta ND, 2013. Lithium in the public water supply and suicide mortality in Texas. J Psychiat Res 47, 407411.

Brahm N, Puls CM, 2011. Hypomania secondary to a change in altitude in an adolescent male. Clin Schizophr Relat Psychoses 5, 102-105.

Brambor T, Clark W, Golder M, 2006. Understanding interaction models: improving empirical analyses. Polit Anal 14, 63-82.

Brenner B, Cheng D, Clark S, Camargo CA, 2011. Positive association between altitude and suicide in 2584 U.S. counties. High Altit Med Biol 12, 31-35.

Fox J, 2002. An R and S-Plus companion to applied regression. Sage, Thousand Oaks.

Fox J, 2003. Effect displays in R for generalised linear models. J Stat Softw 8, 1-27.

Griffith D, 2000. A linear regression solution to the spatial autocorrelation problem. J Geogr Syst 2, 141-156.

Grimpe C, Patuelli R, 2011. Regional knowledge production in nanomaterials: a spatial filtering approach. Ann Regional Sci 46, 519-541.

Grömping U, 2009. Estimators of relative importance in linear regression based on variance decomposition. Am Stat 61, 139147.

Guzzetta F, Tondo L, Centorrino F, Baldessarini RJ, 2007. Lithium treatment reduces suicide risk in recurrent major depressive disorder. J Clin Psychiat 68, 380-383.

Hawton K, van Heeringen K, 2009. Suicide. Lancet 373, 13721381.

Helbich M, Leitner M, Kapusta ND, 2012. Geospatial examination of lithium in drinking water and suicide mortality. Int $\mathrm{J}$ Health Geogr 11, 19.

Huthwaite MA, Stanley J, 2010. Lithium in drinking water. Brit J Psychiat 196, 159-160.

Jaccard J, Teitel L, Turrisi R, 1990. Interaction effects in multi- 
ple regression. Sage, Thousand Oaks.

Kabacs N, Memon A, Obinwa T, Stochl J, Perez J, 2011. Lithium in drinking water and suicide rates across the east of England. Brit J Psychiat 198, 406-407.

Kapusta ND, Mossaheb N, Etzersdorfer E, Hlavin G, Thau K., Willeit M, Praschak-Rieder N, Sonneck G, Leithner-Dziubas $\mathrm{K}, 2011$. Lithium in drinking water and suicide mortality. Brit J Psychiat 198, 346-350.

Kim K, Mickelson JB, Brenner BE, Haws CA, Yurgelun-Todd DA, Renshaw PF, 2011. Altitude, gun ownership, rural areas, and suicide. Am J Psychiat 168, 49-54.

Mann JJ, Apter A, Bertolote J, Beautrais A, Currier D, Haas A, Hegerl U, Lonnqvist J, Malone K, Marusic A, Mehlum L, Patton G, Phillips M, Rutz W, Rihmer Z, Schmidtke A, Shaffer D, Silverman M, Takahashi Y, Varnik A, Wasserman D, Yip P, Hendin H, 2005. Suicide prevention strategies: a systematic review. JAMA 294, 2064-2074.

Ohgami H, Terao T, Shiotsuki I, Ishii N, Iwata N, 2009. Lithium levels in drinking water and risk of suicide. Brit J Psychiat 194, 464-465.

Patuelli R, Griffith D, Tiefelsdorf M, Nijkamp P, 2011. Spatial filtering and eigenvector stability: space-time models for German unemployment data. Int Regional Sci Rev 34, 253-280.

Richardson D, Volkow N, Kwan M-P, Kaplan R, Goodchild M, Croyle R, 2013. Spatial turn in health research. Science 339, 1390-1392.

Schrauzer GN, 2002. Lithium: occurrence, dietary intakes, nutritional essentiality. J Am Coll Nutr 21, 14-21.

Schrauzer GN, Shrestha KP, 1990. Lithium in drinking water and the incidences of crimes, suicides, and arrests related to drug addictions. Biol Trace Elem Res 25, 105-113.

Shvartsev SL, 2008. Geochemistry of fresh groundwater in the main landscape zones of the Earth. Geochem Int 46, 1285 1398.

Thayn J, Simanis J, 2013. Accounting for spatial autocorrelation in linear regression models using spatial wiltering with eigenvectors. Ann Assoc Am Geogr 103, 47-66.

Tiefelsdorf M, Boots B, 1995. The exact distribution of Moran's I. Environ Plann A 27, 985-999.

Tiefelsdorf M, Griffith D, 2007. Semiparametric filtering of spatial autocorrelation: the eigenvector approach. Environ Plann A 39, 1193-1221.

Yang M, 2011. Lithium concentrations in drinking water. Brit J Psychiat 198, 466. 